Linköping Studies in Science and Technology.

Dissertations. No. 1736

\title{
Finite Element Modeling of Contact Problems
}

\author{
Asim Rashid

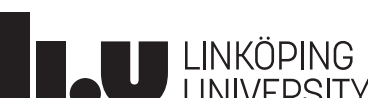

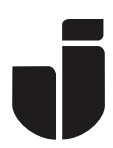 \\ JÖNKÖPING UNIVERSITY \\ School of Engineering
}

Division of Solid Mechanics

Department of Management and Engineering

Linköping University, SE-581 83, Linköping, Sweden

Linköping, January 2016 
Cover:

Nodal contact forces represented as pressure plot on the brake pad surface.

Printed by:

LiU-Tryck, Linköping, Sweden

ISBN 978-91-7685-847-9

ISSN 0345-7524

Distributed by:

Linköping University

Department of Management and Engineering

SE-581 83, Linköping, Sweden

\section{(C) 2016 Asim Rashid}

This document was prepared with $\mathrm{HT}_{\mathrm{E}} \mathrm{X}$, January 20, 2016

No part of this publication may be reproduced, stored in a retrieval system, or be transmitted, in any form or by any means, electronic, mechanical, photocopying, recording, or otherwise, without prior permission of the author. 


\section{Preface}

First of all I would like to express my profound gratitude to my current and previous supervisors; Prof. Peter Hansbo and Prof. Niclas Strömberg, for their support, advice and provisions. I would also like to thank my co-supervisor Prof. Anders Klarbring for his support.

I am thankful to all the colleagues at JTH for a nice working environment. In addition, I would like to thank Sara Kallin and Kent Salomonsson for their valuable comments to improve this manuscript. A special thanks to my former colleague, Magnus Hofwing, for providing the relevant data to the project. I would also like to thank Martin Tapankov for his helpful suggestions for solving Latex related issues and recommending very useful software.

I would also like to express my gratitude to the people at Volvo 3P especially Magnus Levinsson and Per Hasselberg for providing the relevant data and fruitful discussions. Another thanks to Erik Holmberg at Linköping Universtiy for providing the Latex template for this thesis. I am very grateful for the funding by Vinnova, Jönköping University and Volvo 3P.

Finally, I would like to thank my family for their support and patience.

Asim Rashid

Jönköping, 2016-01-18 



\section{Abstract}

Contact is the principal way load is transferred to a body. The study of stresses and deformations arising due to contact interaction of solid bodies is thus of paramount importance in many engineering applications. In this work, problems involving contact interactions are investigated using finite element modeling.

In the first part, a new augmented Lagrangian multiplier method is implemented for the finite element solution of contact problems. In this method, a stabilizing term is added to avoid the instability associated with overconstraining the nonpenetration condition. Numerical examples are presented to show the influence of stabilization term. Furthermore, dependence of error on different parameters is investigated.

In the second part, a disc brake is investigated by modeling the disc in an Eulerian framework which requires significantly lower computational time than the more common Lagrangian framework. Thermal stresses in the brake disc are simulated for a single braking operation as well as for repeated braking. The results predict the presence of residual tensile stresses in the circumferential direction which may cause initiation of radial cracks on the disc surface after a few braking cycles. It is also shown that convex bending of the pad is the major cause of the contact pressure concentration in middle of the pad which results in the appearance of a hot band on the disc surface. A multi-objective optimization study is also performed, where the mass of the back plate, the brake energy and the maximum temperature generated on the disc surface during hard braking are optimized. The results indicate that a brake pad with lowest possible stiffness will result in an optimized solution with regards to all three objectives. Finally, an overview of disc brakes and related phenomena is presented in a literature review.

In the third part, a lower limb donned in a prosthetic socket is investigated. The contact problem is solved between the socket and the limb while taking friction into consideration to determine the contact pressure and resultant internal stress-strain in the soft tissues. Internal mechanical conditions and interface stresses for three different socket designs are compared. Skin, fat, fascia, muscles, large blood vessels and bones are represented separately, which is novel in this work. 



\section{List of Papers}

This thesis is based on the following papers:

I. P. Hansbo, A. Rashid, K. Salomonsson, Least-squares stabilized augmented Lagrangian multiplier method for elastic contact, submitted to Finite Elements in Analysis and Design.

II. A. Rashid, N. Strömberg, An Efficient Sequential Approach for Simulation of Thermal Stresses in Disc Brakes, In the proceedings of the 15th Nordic Symposium on Tribology, NORDTRIB, Trondheim, Norway, 12-15 June, 2012

III. A. Rashid, N. Strömberg, Sequential Simulation of Thermal Stresses in Disc Brakes for Repeated Braking, Journal of Engineering Tribology, 227(8): 919929, 2013.

IV. A. Rashid, N. Strömberg, Thermomechanical Simulation of Wear and Hot Bands in a Disc Brake by adopting an Eulerian approach, In the proceedings of the Eurobrake, Dresden, Germany, 17-19 June, 2013.

V. K. Amouzgar, N. Strömberg, A. Rashid, Multi-Objective Optimization of a Disc Brake System by using SPEA2 and RBFN, In ASME Proceedings | 39th Design Automation Conference: Portland, Oregon, USA, August 4-7, 2013.

VI. A. Rashid. Overview of Disc Brakes and Related Phenomena - a literature review, International Journal of Vehicle Noise and Vibration, 10(4): 257-301, 2014.

VII. A. Rashid, S. Kallin, K. Salomonsson, P. Hansbo, Simulation of internal mechanical conditions in the lower limb donned in a transtibial prosthetic socket, submitted to Journal of Biomechanics.

Papers have been reformatted to fit the layout of the thesis. 


\section{Contributions to co-authored papers}

N. Strömberg developed the toolbox, which was used for frictional heat transfer analysis in all papers concerning the disc brake.

\section{Paper I.}

Prepared a numerical example.

Wrote about the numerical results in the paper.

\section{Paper II.}

Carried out the numerical simulations.

Predominantly responsible for planning and writing the paper.

\section{Paper III.}

Carried out the numerical simulations.

Predominantly responsible for planning and writing the paper.

\section{Paper IV.}

Carried out the numerical simulations.

Predominantly responsible for planning and writing the paper.

\section{Paper VI.}

Prepared FE model for optimization study.

Wrote about the disc brake problem in the paper.

\section{Paper VII.}

Carried out the numerical simulations in consultation with other authors.

Predominantly responsible for planning and writing the paper. 


\section{Contents}

$\begin{array}{ll}\text { Preface } & \text { iii }\end{array}$

Abstract $\quad$ v

List of Papers vii

Contents $\quad$ ix

1 Introduction 1

1.1 Background . . . . . . . . . . . . . . . . . . 1

1.1.1 Stabilized augmented Lagrangian multiplier method . . . . . 2

1.1.2 Disc brakes . . . . . . . . . . . . . . . . 2

1.1.3 Prosthetic sockets . . . . . . . . . . . . . . . 3

2 Contact mechanics 5

2.1 Introduction . . . . . . . . . . . . . . . . . 5

2.1.1 Enforcement of contact constraints . . . . . . . . . 7

3 Disc brakes $\quad 9$

3.1 Background ........................ . . 9

3.2 Governing equations . . . . . . . . . . . . . . . . 12

3.2.1 Heat transfer analysis . . . . . . . . . . . . . . . . 12

3.2 .2 Stress analysis . . . . . . . . . . . . . . . 13

3.3 Material model . . . . . . . . . . . . . . . . . . . . . . . . . . 17

3.4 Residual stresses . . . . . . . . . . . . . . . . . . . . . 17

3.4.1 A simple example . . . . . . . . . . . . . . . . . . 18

3.5 Results for the disc-pad system . . . . . . . . . . . . . . . . 21

4 Prosthetic sockets $\quad 27$

4.1 Background . . . . . . . . . . . . . . . . . 27

5 Review of included papers $\quad 29$

$\begin{array}{ll}\text { Bibliography } & 34\end{array}$

$\begin{array}{ll}\text { Paper I } & 37\end{array}$ 
Paper II

Paper III

Paper IV

Paper V

113

Paper VI

133

Paper VII 


\section{Introduction}

\subsection{Background}

The study of stresses and deformations arising from the interaction of solid bodies at their surfaces is of paramount importance in many engineering applications. The understanding of these contact interactions is crucial for the design of safe, comfortable, energy efficient and long lasting products, e.g. disc brakes, prosthetic sockets, seat cushions, tires, piston rings, bearings and clutches. In many applications, tribological interactions of contacting bodies alter the topography of mating surfaces over time. This makes the investigation of contact problems very challenging.

Analytical solutions for the contact problems have been proposed for contacting bodies with fairly simple geometries and loading conditions. However, in many industrial applications the contact problem is highly complex due to intricate geometries of contacting bodies, large deformations and nonlinear materials. Extension of the analytical solutions to such problems is even difficult to contemplate. $\mathrm{Nu}$ merical solution techniques for contact problems have evolved significantly over the decades and can solve even complex engineering problems. Furthermore, numerical implementation allows to take into account the changing contact conditions due to thermal deformations and wear of contacting bodies.

The finite element method (FEM) and boundary element method (BEM) have been used for the numerical solution of contact problems [1]. FEM is generally used for solving the contact problems in solid mechanics [2]. Many commercial finite element software packages possess the capability to solve contact problems. The methodologies available in these packages for solving contact interactions are relatively immature in comparison to the capabilities in the general area of nonlinear solid mechanics [3]. This is mainly due to the reason that the contact problems are highly nonlinear. Intensive research is still going on to develop robust contact algorithms [4].

In the present thesis, a new stabilized augmented Lagrangian multiplier method is implemented for the finite element solution of contact problems. Furthermore, contact problems of a disc brake and a lower limb donned in a prosthetic socket are investigated using finite element analysis. 


\subsubsection{Stabilized augmented Lagrangian multiplier method}

Enforcement of Signorini contact conditions is an important consideration to obtain a solution for a contact problem. Many different methods are available for that purpose, e.g. the Lagrange multiplier method, the penalty method and the augmented Lagrangian multiplier method. Among these the augmented Lagrangian method, which is a combination of the penalty and the Lagrangian multiplier methods, is widely used as it facilitates the iterative procedures for the contact problem solution. Like the Lagrange multiplier method, the augmented Lagrange method requires careful matching of approximation of contact pressure (the multiplier) and displacement fields, unless some kind of stabilization is introduced. In this work a new stabilized augmented Lagrangian multiplier method is implemented for the finite element solution of contact problems and presented in Paper I.

\subsubsection{Disc brakes}

Disc brakes are an important component of a vehicle retardation system. They are used to stop or adjust the speed of a vehicle with changing road and traffic conditions. During braking, a set of pads is pressed against a rotating disc. Due to friction, heat is generated at the disc-pad interface, which causes the disc surface temperature to rise in a short period of time. Thermomechanical deformations and wear of the pad makes it a challenging contact problem.

Today, the prevalent way to simulate the thermomechanical behavior of disc brakes in commercial finite element analysis (FEA) software is to use the fully coupled Lagrangian approach in which the finite element mesh of a disc rotates relative to a brake pad and, thermal and mechanical analyses are performed simultaneously. Although this approach works well, it is not feasible due to extremely long computational times. The main reason is the relative sliding of the disc which requires a considerable computational effort to solve the contact problem as the disc rotates.

In this work, the brake disc is modelled using an Eulerian approach, in which the finite element mesh of the disc does not rotate relative to the brake pad but the material moves through the mesh. This requires significantly lower computational time as compared to the Lagrangian approach. Because the finite element mesh of the disc does not rotate relative to the pad, the contact region is always well defined relative to the mesh. This allows the mesh to be refined only in the region where the brake pad is in contact with the disc, which also results in lower computational time.

The Eulerian approach has been used to simulate thermal stresses in a brake disc for a single braking operation as well for repeated braking. Results of these studies are presented in Paper II and III, respectively. In Paper IV, the influence of the wear history of a pad on the temperature distribution on a disc surface is presented. Results for a multi-objective optimization study are presented in Paper V where the 
mass of the back plate, the brake energy and the maximum temperature generated on the disc surface during hard braking are optimized. An overview of disc brakes and related phenomena is presented as a literature review in Paper VI.

\subsubsection{Prosthetic sockets}

Biomechanical understanding of the interaction between a residual limb and a prosthetic socket is very important for designing a comfortable socket. Despite the improved designs of prosthetic sockets, skin problems are reported by a considerable number of lower limb amputees [34]. In addition to superficial skin problems, a subject using a prosthetic socket can also suffer from deep tissue injury [35].

Internal mechanical conditions in the lower limb under external compression have been investigated using FEA [37, 38, 39]. One major concern about the previous studies is that soft tissues have been lumped together to simplify the model. Another concern is that in these studies, displacements or contact pressure have been determined by physical experiments and used as a boundary condition in numerical computations. Although such studies give valuable insight, for the design practice of prosthetic sockets, contact analysis should be performed to determine the boundary conditions.

This work aims to compare the internal mechanical conditions and interface stresses for three different socket designs. Skin, fat, fascia, muscles, blood vessels and bones are represented separately, which is novel in this research. The contact problem is solved between socket and limb while taking friction into consideration to determine the contact pressure and resultant internal stress-strain in the soft tissues. Results for this study are presented in Paper VII.

\section{Outline}

The thesis is organized as follows. First a short introduction is given to the field of contact mechanics. Then a background to the problem of disc brakes is presented and residual stresses are explained with the help of a simple example. Finally, a background to prosthetic sockets is presented. 



\section{Contact mechanics}

\subsection{Introduction}

Contact mechanics is the study of stresses and deformations arising due to the interaction of solid bodies at their surfaces. Contact is a principal way of transferring load to a body and hence problems involving contact interactions are widespread in engineering applications. The tribological interactions of contacting bodies may cause the loss of material from the surfaces. The experimental study of such tribological interfaces is quite complex as different mechanisms come into play at the contacting surfaces at different scales. Friction and wear are the most important characteristics to describe the contacting surfaces. The representation of these characteristics in the form of a mathematical model is very important for the numerical solution of a contact problem. The goal of mathematical models is to have a good agreement between predicted behavior and experimental observations. Usually, there are some simplifications involved in the development of such models. The numerical solution of contact problems which depend on these mathematical models thus also imply simplifications.

The discipline of contact mechanics is mainly built on the subjects of continuum mechanics and mechanics of materials. FEM is generally used for solving the contact problems in solid mechanics [2]. Therefore basic understanding of these subjects is crucial for developing methods for the numerical solution of contact problems.

Contact problems, for the purpose of numerical treatment, can be subdivided into two groups: one body contact problem, and two body contact problem. In the one body contact problem, also known as Signorini problem, a deformable body contacts a rigid obstacle. In the two body contact problem, both bodies involved in contact are deformable bodies.

We consider an elastic body $\Omega$ in $\mathbb{R}^{2}$, with boundary $\Gamma=\Gamma^{\mathrm{D}} \cup \Gamma^{\mathrm{N}} \cup \Gamma^{\mathrm{C}}$ and outward pointing unit normal vector $\boldsymbol{n}$. First, we consider a case where the domain is subjected to volume forces $\boldsymbol{f}$, prescribed boundary displacements on $\Gamma^{\mathrm{D}}$ and tractions on $\Gamma^{\mathrm{N}}$. The boundary value problem in linear elasticity consists in finding 
the displacement field $\boldsymbol{u}$ satisfying the equations and conditions

$$
\begin{aligned}
-\operatorname{div} \boldsymbol{\sigma} & =\boldsymbol{f} & \text { in } \Omega, \\
\boldsymbol{\sigma} & =\boldsymbol{D} \boldsymbol{r} & \text { in } \Omega, \\
\boldsymbol{u} & =\mathbf{0} & \text { on } \Gamma^{\mathrm{D}}, \\
\boldsymbol{\sigma} \cdot \boldsymbol{n} & =\boldsymbol{t} & \text { on } \Gamma^{\mathrm{N}},
\end{aligned}
$$

where $\boldsymbol{\sigma}$ is the stress tensor, $\boldsymbol{D}$ is the elasticity tensor and $\boldsymbol{\varepsilon}=\boldsymbol{\varepsilon}(\boldsymbol{u})$ is the small strain tensor. The weak or variational form of the above problem is given as follows: find $\boldsymbol{u}$ such that $\boldsymbol{u}=\mathbf{0}$ on $\Gamma^{\mathrm{D}}$ and

$$
\int_{\Omega} \boldsymbol{\sigma}(\boldsymbol{u}): \boldsymbol{\varepsilon}(\boldsymbol{v}) \mathrm{d} \Omega=\int_{\Omega} \boldsymbol{f} \cdot \boldsymbol{v} \mathrm{d} \Omega+\int_{\Gamma^{\mathrm{N}}} \boldsymbol{t} \cdot \boldsymbol{v} \mathrm{ds}
$$

for all admissible weighting functions $\boldsymbol{v}$. Here the admissible functions are the ones satisfying $\boldsymbol{v}=\mathbf{0}$ on $\Gamma^{\mathrm{D}}$. In this problem displacements and tractions that are prescribed on the boundaries are known a priori and also the the areas on which they act.

Now we consider a case when the elastic body comes into frictionless contact with a rigid obstacle along $\Gamma^{\mathrm{C}}$ as shown in figure 1. This gives rise to normal traction on the body surface. The boundary value problem consists of finding the displacement field $\boldsymbol{u}$ and normal traction $\sigma_{n}(\boldsymbol{u})$ acting on body along $\Gamma^{\mathrm{C}}$. The weak or variational form of the contact problem can be written as follows: find $\boldsymbol{u}$ and $\sigma_{n}(\boldsymbol{u})$ such that $\boldsymbol{u}=\mathbf{0}$ on $\Gamma^{\mathrm{D}}$ and

$$
\int_{\Omega} \boldsymbol{\sigma}(\boldsymbol{u}): \boldsymbol{\varepsilon}(\boldsymbol{v}) \mathrm{d} \Omega=\int_{\Omega} \boldsymbol{f} \cdot \boldsymbol{v} \mathrm{d} \Omega+\int_{\Gamma^{\mathrm{N}}} \boldsymbol{t} \cdot \boldsymbol{v} \mathrm{ds}+\int_{\Gamma^{\mathrm{C}}} \sigma_{n}(\boldsymbol{u}) \boldsymbol{v} \cdot \boldsymbol{n} \mathrm{ds}
$$

for all admissible $\boldsymbol{v}$. In this new boundary value problem, contact traction, displacement field and area on which traction acts need to be determined as part of solution. The solution can be found by introducing the following conditions governing the unilateral contact,

$$
\begin{array}{r}
g \leq 0 \\
\sigma_{n} \leq 0 \\
g \sigma_{n}=0
\end{array}
$$

where $g:=u_{n}-g_{0}, \sigma_{n}(\boldsymbol{u}):=\boldsymbol{n} \cdot \boldsymbol{\sigma}(\boldsymbol{u}) \cdot \boldsymbol{n}$ and $u_{n}:=\boldsymbol{u} \cdot \boldsymbol{n}$. The initial gap, $g_{0}:=\left[\boldsymbol{x}-\boldsymbol{x}_{\mathbf{0}}\right] \cdot \boldsymbol{n}$, is the gap at zero displacement between a point $\boldsymbol{x}$ of elastic body and a corresponding point $\boldsymbol{x}_{\mathbf{0}}$ of rigid obstacle which are coming into contact. 
These contact constraints are known as Hertz-Signorini-Moreau conditions or simply Signorini conditions in the literature [5]. The first condition makes sure that the penetration of contacting bodies does not take place. The second condition describes that the normal traction can only be compressive (ignoring the tensile stress due to molecular attraction between bodies which is negligible) and the third condition corresponds to the fact that the contact traction only becomes non-zero when contact is established.

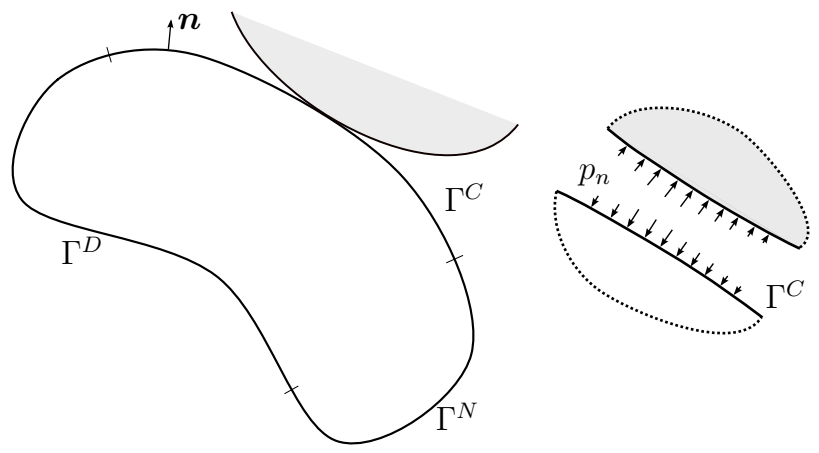

Figure 1: A deformable body in frictionless contact with a rigid obstacle.

\subsubsection{Enforcement of contact constraints}

Several methods are available to incorporate the contact constraints (4) into the variational formulation, e.g. the Lagrange multiplier method, the penalty method, the augmented Lagrangian multiplier method and the Nitsche method. First three will be described here briefly.

Lagrange multiplier method

This method [6] can be formulated as follows: find $\boldsymbol{u}$ and $p_{n}$ such that

$$
\begin{aligned}
& \int_{\Omega} \boldsymbol{\sigma}(\boldsymbol{u}): \boldsymbol{\varepsilon}(\boldsymbol{v}) \mathrm{d} \Omega=\int_{\Omega} \boldsymbol{f} \cdot \boldsymbol{v} \mathrm{d} \Omega+\int_{\Gamma^{\mathrm{N}}} \boldsymbol{t} \cdot \boldsymbol{v} \mathrm{ds}+\int_{\Gamma^{\mathrm{C}}} p_{n} \boldsymbol{v} \cdot \boldsymbol{n} \mathrm{ds} \\
& \int_{\Gamma^{\mathrm{C}}} g q \mathrm{ds}=0
\end{aligned}
$$

for all admissible $\boldsymbol{v}$ and $q$. Here $p_{n}$ is the Lagrange multiplier (the contact pressure) replacing $\sigma_{n}$. The multipliers are only active when contact is established. 
Penalty method

The penalty method works by penalizing the violation of the contact constraint. In this method, $\sigma_{n}$ is replaced by $p_{n}$ (the contact pressure) where $p_{n}=-\frac{1}{\gamma}[g]_{+}$. Here $\gamma \gg 0$ is a given penalty parameter and

$$
[x]_{+}=\frac{x+|x|}{2}
$$

The weak form for the contact problem can be written as: find $\boldsymbol{u}$ such that

$$
\int_{\Omega} \boldsymbol{\sigma}(\boldsymbol{u}): \boldsymbol{\varepsilon}(\boldsymbol{v}) \mathrm{d} \Omega=\int_{\Omega} \boldsymbol{f} \cdot \boldsymbol{v} \mathrm{d} \Omega+\int_{\Gamma^{\mathrm{N}}} \boldsymbol{t} \cdot \boldsymbol{v} \mathrm{ds}-\int_{\Gamma^{\mathrm{C}}} \frac{1}{\gamma}[g]_{+} \boldsymbol{v} \cdot \boldsymbol{n} \mathrm{ds}
$$

for all admissible $\boldsymbol{v}$. The penalty method leads to a problem to be solved only in one variable, $\boldsymbol{u}$, instead of two as in (3).

Augmented Lagrangian method

The augmented Lagrangian approach combines the penalty and the Lagrangian multiplier methods. In this method, $\sigma_{n}$ is replaced by $p_{n}=-\frac{1}{\gamma}\left[g-\gamma p_{n}\right]_{+}$. The weak form for the contact problem can be written as: find $\boldsymbol{u}$ and $p_{n}$ such that

$$
\int_{\Omega} \boldsymbol{\sigma}(\boldsymbol{u}): \boldsymbol{\varepsilon}(\boldsymbol{v}) \mathrm{d} \Omega+\int_{\Gamma^{\mathrm{C}}} \frac{1}{\gamma}\left[g-\gamma p_{n}\right]_{+}(\boldsymbol{v} \cdot \boldsymbol{n}-\gamma q) \mathrm{ds}-\int_{\Gamma^{\mathrm{C}}} \gamma p_{n} q \mathrm{ds}=\int_{\Omega} \boldsymbol{f} \cdot \boldsymbol{v} \mathrm{d} \Omega+\int_{\Gamma^{\mathrm{N}}} \boldsymbol{t} \cdot \boldsymbol{v} \mathrm{ds}
$$

for all admissible $\boldsymbol{v}$ and $q$. The statement $p_{n}=-\frac{1}{\gamma}\left[g-\gamma p_{n}\right]_{+}$is equivalent to the contact constraints (4) as described in Paper I. This, however, is not a stabilized method and requires careful matching of approximation of contact pressure and displacement fields for the stability of the discrete problem. The other option is to introduce some kind of stabilization. In this work a new stabilized augmented Lagrangian multiplier method is implemented for the finite element solution of contact problems and presented in Paper I. 


\section{Disc brakes}

\subsection{Background}

Disc brakes are an important component of a vehicle retardation system. They are used to stop or retard a vehicle with changing road and traffic conditions. During braking, a set of pads is pressed against a rotating disc and, due to friction, heat is generated at the disc-pad interface, which must be dispersed. The heat causes the disc surface temperature to rise in a short period of time. This heat ultimately transfers to the vehicle and the environment, and the disc cools down.

As a result of higher temperatures, in addition to local changes of the contact surfaces, there are global deformations occurring in the disc and the pad. Due to different geometries of discs, each disc has different geometrical constraints to the thermal expansion and so the deformations can appear in different forms in different discs. Some of the most commonly observed thermal deformations are coning and buckling $[7,8,9,10,11]$. Such geometrical deviations could be avoided or reduced if the thermal loading and disc geometry are symmetric about the midplane of the disc [12, 13], and the friction ring is decoupled from the mounting bell so that it has relatively more freedom to expand in the radial direction [14]. This is usually attempted to achieve by using a so-called composite brake disc in which mounting bell and friction ring are separated from each other. Such a composite brake disc is shown in figure 2 .

In addition to these deformations, macrocracks might also appear on a disc surface in the radial direction after a number of brake cycles, affecting the performance and life of a brake disc $[16,17]$. It has been shown in many previous works, e.g. $[10,18,19]$, that during hard braking, high compressive stresses are generated in the circumferential direction on the disc surface which cause plastic yielding. But when the disc cools down, these compressive stresses transform to tensile stresses. For repeated braking when this kind of stress-strain history is repeated, stress cycles with high amplitudes are developed which might generate low cycle fatigue cracks after a few braking cycles. Dufrénoy and Weichert [10], confirmed the existence of residual tensile stresses on the disc surface by measuring with the hole drilling strain gage method. In the present work investigation of the stresses which cause these cracks on a disc surface, by using finite element simulations, is a major focus. 


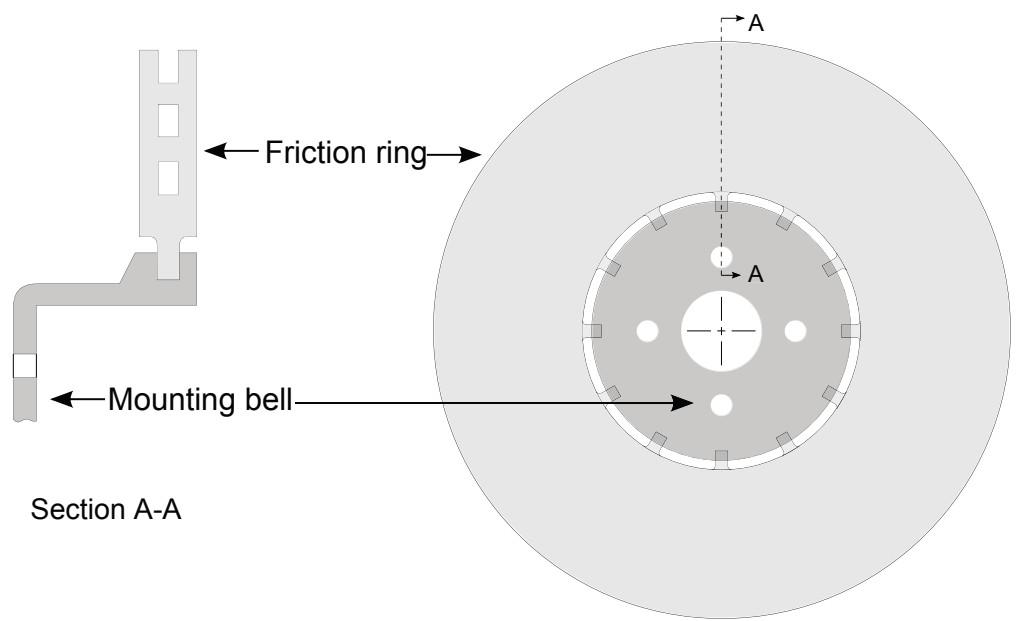

Figure 2: Simplified representation of a composite brake disc showing an integrally casted mounting bell with a friction ring [15].

Many researchers have used FEA techniques to predict the thermomechanical behavior of disc brakes. To simplify the development of a FEA model for a solid disc (as compared to a ventilated disc) it is often assumed that the pad is smeared over the entire $360^{\circ}$, implying that the disc-pad system can be considered axisymmetric, see e.g. $[20,21]$. In this simplified model, circumferential variation of temperature and contact pressure cannot be predicted. Another approach to simplify the model for a ventilated disc is to consider only a small sector of a disc by taking rotational symmetry into account, see e.g. [9, 10]. Again the assumption has to be made about the smearing of the pad, implying that circumferential variations of temperature and contact pressure cannot be predicted satisfactorily. It has been shown in previous work $[22,23]$ that a pad also undergoes thermal deformation, called convex bending; furthermore temperature distribution is not constant along the circumference of a disc [24]. Thus, it is clear that these approaches are not sufficient to model the real behavior, instead a FEA model with complete three dimensional (3D) geometries of a disc and pads is required. Some researchers, see e.g. [7, 18], have indeed used complete $3 \mathrm{D}$ geometries to determine the thermomechanical behavior of disc brakes realistically.

Today, the prevalent way to simulate frictional heating of disc brakes in commercial software is to use the fully coupled Lagrangian approach in which the finite element mesh of a disc rotates relative to a brake pad and thermal and mechanical analysis is performed simultaneously. Although this approach works well, it is not feasible due to extremely long computational times. Particularly, for simulating repeated braking, this approach is not feasible for practical use. As a brake disc could be considered a solid of revolution, partially or fully, it is possible to model it using an Eulerian approach, in which the finite element mesh of the disc does not rotate 

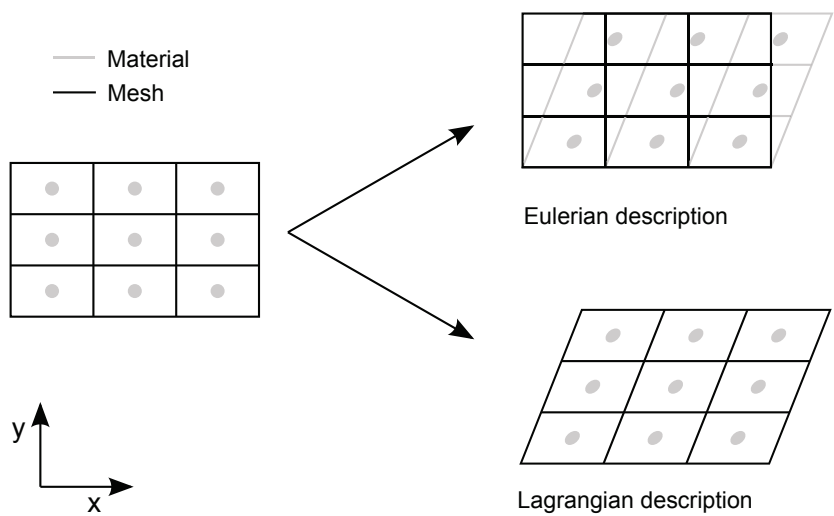

Figure 3: Schematic representation of the Eulerian and the Lagrangian approaches.

relative to the brake pad but the material moves through the mesh. This requires significantly lower computational time as compared to the Lagrangian approach. Figure 3 shows schematically both the Eulerian and the Lagrangian approaches.

The simulations performed within this work are by using a sequential approach where temperature history from the frictional heat analysis is used as an input in a coupled stress analysis. The frictional heat analysis, based on the Eulerian method, is performed in an in-house software developed by Strömberg, which is described in his earlier works $[25,26]$. The Eulerian approach gives rise to a convective velocity term in the equation of motion. If the speed of convection is high, some kind of stabilization is required. In the in-house software a streamline-upwind approach is adapted to stabilize the solution [26]. In this implementation, the contact pressure is not constant, but varies at each time step taking into account the thermomechanical deformations of the disc and the pad. This updated contact pressure information is used to compute heat generation and flow to the contacting bodies at each time step. In such manner, the nodal temperatures are updated accurately and their history is recorded at each time step. Later stress analysis is performed in the commercial software Abaqus, which uses thermal history from the frictional heat analysis as an input. Figure 4 shows the workflow of this sequential approach schematically. Stresses due to the applied normal brake force, centrifugal forces and deceleration forces are insignificant in comparison to the thermal stresses [27] so only thermal stresses are considered in this work.

The results show that during hard braking high compressive stresses are generated on the disc surface in the circumferential direction which cause yielding. But when the disc cools down, these compressive stresses transform to tensile residual stresses. For repeated braking an approximately stable stress-strain loop is obtained. So, if the fatigue life data for the disc material is known, its fatigue life can be assessed. It is also shown that convex bending of the pad due to thermal deformations is the major cause of contact pressure concentration and hence appearance of hot 


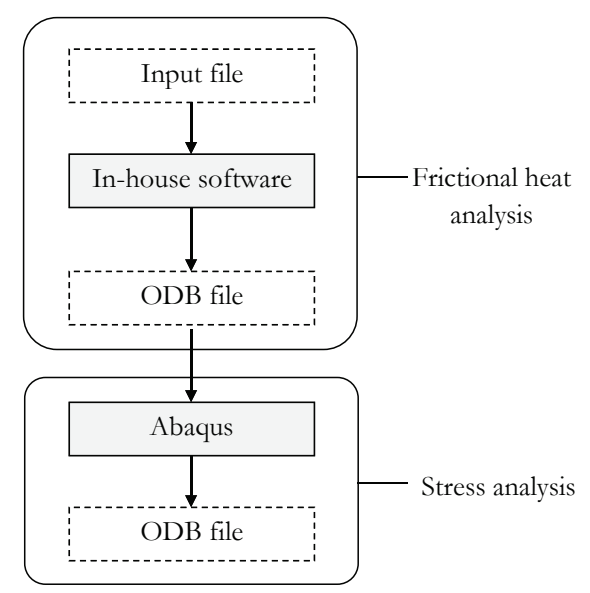

Figure 4: Workflow of the sequential approach to determine the thermal stresses.

bands. The results show that when wear is considered, different distributions of temperature on the disc surface are obtained for each new brake cycle. After a few braking cycles two hot bands appear on the disc surface instead of only one. These results are in agreement with experimental observations. The sequential approach requires significantly lower computational time as compared to the Lagrangian approach which makes it possible to perform multi-objective optimization studies. Preliminary results of such a study are also presented in this work.

\subsection{Governing equations}

The governing equations for the frictional heat analysis, and stress analysis while employing two different material hardening models will be described here.

\subsubsection{Heat transfer analysis}

Frictional heat power generated at the contact interface of a disc-pad system can be expressed as

$$
q_{\text {gen }}=\mu p_{n} \omega r
$$

where $\mu$ is the coefficient of friction, $r$ is the distance of a contact pair from the center of the disc, $p_{n}$ is the normal component of the contact traction vector, and $\omega$ is the angular velocity of the disc. The frictional heat generated at the contact interface flows into the disc and the pad. Heat conduction for each body is governed 
by the classical heat equation

$$
\rho c \dot{T}=k \sum_{i=1}^{3} \frac{\partial^{2} T}{\partial x_{i}^{2}},
$$

where $\rho$ is the density, $c$ is the specific heat capacity and $k$ is the thermal conductivity. For the disc the material time derivative is defined by

$$
\dot{T}=\frac{\partial T}{\partial t}+\nabla T \cdot \boldsymbol{v}
$$

where $\boldsymbol{v}$ is the convective velocity due to the Eulerian framework.

\subsubsection{Stress analysis}

Stress-strain relations used to describe deformation of a material are different for the elastic and plastic domain. Consequently, it is important to know if the stress state is in the elastic or plastic domain. For this purpose a yield criterion is used to suggest the limit of elasticity and the initiation of yielding in a material under any combination of stresses. There are several yield criteria used in practice. Some of these are: the maximum shear stress criterion, the maximum principal stress criterion and the von Mises stress criterion. These criteria could be expressed in terms of material constants obtained from different physical tests, e.g. a shear or a uniaxial tensile test. In this work these material parameters are obtained by considering uniaxial tests at different temperatures.

According to the von Mises stress criterion, yielding depends on the deviatoric stress and not the hydrostatic stress. It is expressed as

$$
\begin{array}{ll}
\sqrt{3 J_{2}}-\sigma_{y}=0 & \text { for plastic deformation } \\
\sqrt{3 J_{2}}-\sigma_{y}<0 & \text { for elastic deformation }
\end{array}
$$

where $\sigma_{y}$ is the stress at yield in a uniaxial test and $J_{2}$ is the second invariant of the deviatoric stress, i.e.

$$
J_{2}=\frac{1}{2} s: s
$$

where $s$ is the deviatoric stress, given by

$$
\boldsymbol{s}=\boldsymbol{\sigma}-\frac{\operatorname{tr}(\boldsymbol{\sigma})}{3} \boldsymbol{I} .
$$




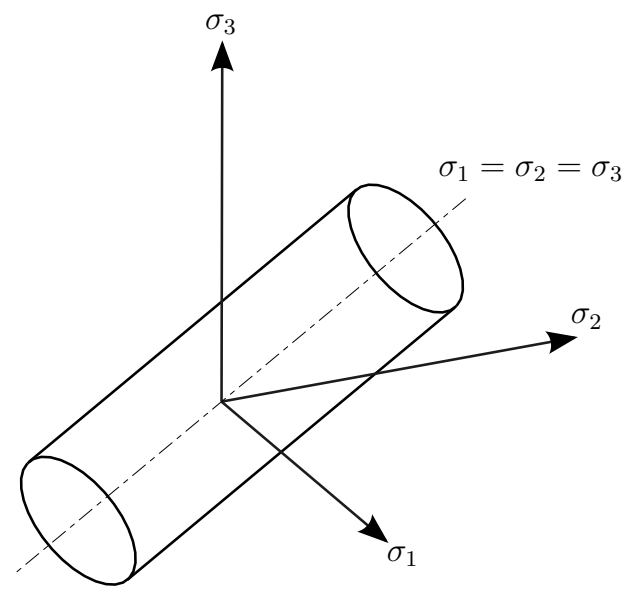

Figure 5: Schematic of the von Mises yield surface in the principal stress space.

The von Mises yield criterion appears as a cylindrical surface in the principal stress space as shown in figure 5. A loading case where stresses lie inside this surface is said to be an elastic loading. The yield surface can be described as a boundary between elastic and plastic deformation regions.

During plastic deformations, the yield surface can translate, expand or distort in the stress space [28]. Two models are frequently used to describe the hardening behavior of a material due to plastic deformations: isotropic hardening and kinematic hardening. Isotropic hardening assumes that the yield surface expands uniformly as shown in figure 6a. Kinematic hardening assumes that the yield surface translates in the stress space as shown in figure 6b. Pure isotropic hardening cannot predict the Bauschinger effect, as shown for a uniaxial loading case in figure 7 , which has been observed in many materials experimentally. In general, neither the isotropic nor the kinematic hardening model truly represents the real material hardening behavior which could be quite complicated [28].

Given the temperature history, thermal strains are determined according to

$$
\varepsilon^{t}=\alpha(T)\left(T-T_{\text {ref }}\right)-\alpha\left(T_{i}\right)\left(T_{i}-T_{\text {ref }}\right),
$$

where $\alpha(T)$ is the thermal dilatation coefficient, $T_{\text {ref }}$ is a reference temperature and $T_{i}$ is the initial temperature. The infinitesimal strain $\varepsilon$ is split into elastic, plastic and thermal strains, expressed as

$$
\varepsilon=\varepsilon^{e}+\varepsilon^{p}+\varepsilon^{t},
$$

where $\varepsilon^{e}$ and $\varepsilon^{p}$ represent the elastic and plastic strains, respectively. $\varepsilon^{e}$ is determined from this relation and then stresses can be computed by using Hooke's law as

$$
\boldsymbol{\sigma}=\boldsymbol{D} \varepsilon^{e}
$$




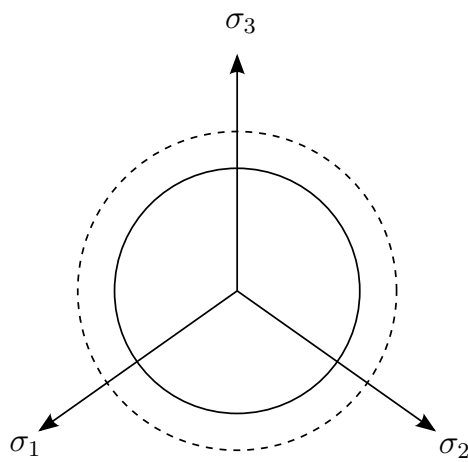

(a) Isotropic hardening

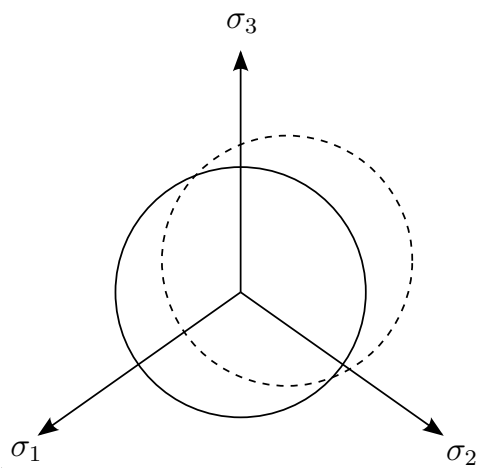

(b) Kinematic hardening

Figure 6: Evolution of the von Mises yield surface with isotropic and kinematic hardening. Solid line represents initial yield surface and dashed line represents subsequent yield surface.

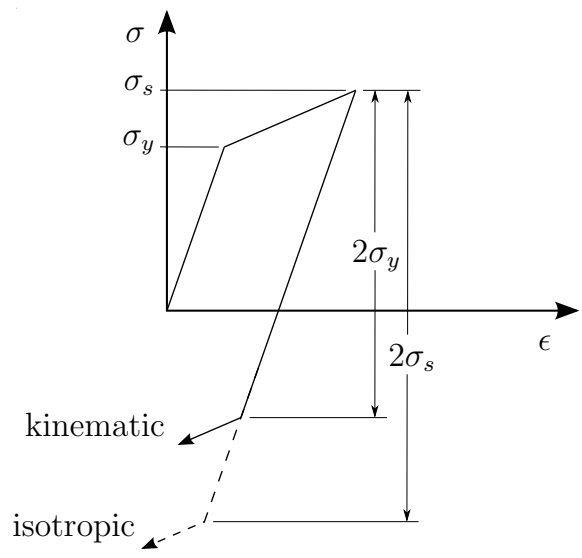

Figure 7: Uniaxial stress-strain curves for isotropic and kinematic hardening. 
where $\boldsymbol{D}$ is the elasticity tensor. The stresses satisfy the following equilibrium equation:

$$
\operatorname{div}(\boldsymbol{\sigma})=\mathbf{0}
$$

When the von Mises yield criterion with isotropic hardening model is used, the yield surface is defined as

$$
f\left(\boldsymbol{\sigma}, \varepsilon^{p}, T\right)=\sqrt{3 J_{2}}-\sigma_{y}-K,
$$

where $\sigma_{y}=\sigma_{y}(T)$ is the uniaxial yield strength and $K=K\left(\varepsilon_{\mathrm{eff}}^{p}, T\right)$ is the hardening parameter. The effective plastic strain $\varepsilon_{\text {eff }}^{p}$ is expressed as

$$
\varepsilon_{\mathrm{eff}}^{p}=\int_{0}^{t} \sqrt{\frac{2 \dot{\varepsilon}^{p}: \dot{\varepsilon}^{p}}{3}} d t .
$$

The plastic strain $\varepsilon^{p}$ is governed by the following associative law

$$
\dot{\varepsilon}^{p}=\dot{\lambda} \frac{3 s}{2 \sqrt{3 J_{2}}},
$$

where $\lambda$ is the plastic multiplier, which is determined by the Karush-Kuhn-Tucker conditions:

$$
\dot{\lambda} \geq 0, \quad f \leq 0, \quad \dot{\lambda} f=0 .
$$

When the von Mises yield criterion with kinematic hardening model is used, the yield surface is defined as

$$
f(\boldsymbol{\eta}, T)=\sqrt{\frac{3}{2} \boldsymbol{\eta}: \boldsymbol{\eta}}-\sigma_{y},
$$

where

$$
\eta=s-\alpha
$$

and $\boldsymbol{\alpha}$ is the back-stress tensor. The evolution of the back-stress is governed by Ziegler's rule, which can be written as

$$
\dot{\boldsymbol{\alpha}}=\frac{k_{h}}{\sigma_{y}}(\boldsymbol{s}-\boldsymbol{\alpha}) \dot{\varepsilon}_{\mathrm{eff}}^{p},
$$

where $k_{h}=k_{h}(T)$ is the kinematic hardening modulus and the plastic strain $\varepsilon^{p}$ is governed by the following associative law:

$$
\dot{\boldsymbol{\varepsilon}}^{p}=\dot{\lambda} \frac{3}{2} \frac{\boldsymbol{\eta}}{\sqrt{\frac{3}{2} \boldsymbol{\eta}: \boldsymbol{\eta}}} .
$$




\subsection{Material model}

To predict the thermomechanical behavior of a component realistically, it is important to have a material model which represents its characteristics sufficiently accurately. During the frictional heat analysis, temperature independent material data has been used for all the components. For more realistic results, temperature dependent material data should be used. During stress analysis only the brake disc is considered. The brake disc is casted in a grey iron alloy. The material model used in the present work was developed in an earlier work [29] in order to simulate residual stresses in castings from solidification and is now utilized for thermomechanical stress analysis.

Most of the material parameters required to develop this model were obtained from measurements. Young's modulus, the yield strength and hardening behavior were obtained from tensile tests performed at $20^{\circ} \mathrm{C}, 200^{\circ} \mathrm{C}, 400^{\circ} \mathrm{C}, 600^{\circ} \mathrm{C}$ and $800^{\circ} \mathrm{C}$. The data was assumed or collected from literature for temperatures above $800^{\circ} \mathrm{C}$. This material data is used to build a temperature dependent material model with nonlinear hardening which is described in detail in Paper II. The same data is used to build a temperature dependent material model with linear hardening by connecting the first and last point of the hardening curve with a straight line. This linear hardening model is described in detail in Paper III.

The grey iron alloy shows different yield properties in tension and compression [13]. In the present work, it is assumed that the material has the same behavior both in tension and compression. Although this assumption is unrealistic, it is not the purpose of this work to develop a better material model. Moreover, in this work, the von Mises yield criterion is used both in tension and compression.

In [13] a material model which employs the maximum principal stress yield criterion in tension and von Mises yield criterion in compression was used. Another material model which considers different yield behaviors in tension and compression, and employs the von Mises yield criterion both in tension and compression, is reported in [13] and [9]. In the latter model, the numerical results were much closer to the measured experimental data.

\subsection{Residual stresses}

Sometimes, permanent stresses develop in a component even after the external cause, e.g. heat gradient or force, has been removed. In the case of thermal stresses, if they are sufficiently large to cause yielding in a component, then these stresses may develop to residual stresses when the component cools down. Residual stresses may affect the behavior and life of such a component. 


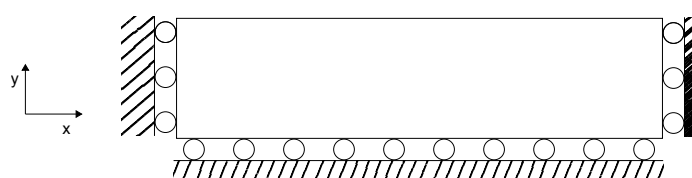

Figure 8: The bar considered for the example shown with boundary conditions.

\subsubsection{A simple example}

In order to describe this kind of phenomena, finite element analysis of a bar subjected to a temperature load will be described and presence of residual stresses after the cooling of the bar will be shown.

In figure 8, a bar is shown with boundary conditions. This bar is subjected to a cyclic temperature load as shown in figure 9, where the peak temperature is $T_{m}=600^{\circ} \mathrm{C}$. The analysis is performed in Abaqus where the bar is meshed with 8 -node biquadratic plane stress quadrilateral elements and reduced integration is used. The material models described in section 3.3 will be used to compute stresses in the bar.

In figure 10, a graph of different strain measures in the longitudinal direction is shown for only one load cycle while employing the temperature independent material model with linear isotropic hardening. It can be seen that the thermal strain increases linearly with the temperature increase. As the bar is restrained in the longitudinal direction, the expanding material causes compressive strain in the bar. In the beginning only compressive elastic strains appear but as the material reaches the elastic limit, compressive plastic yielding also starts. Both the elastic and the plastic strains keep on increasing as the thermal strain increases. After the thermal strain starts decreasing, the elastic strain first shows decreasing trend and later becomes tensile in nature. During this decrease and reversal of the elastic

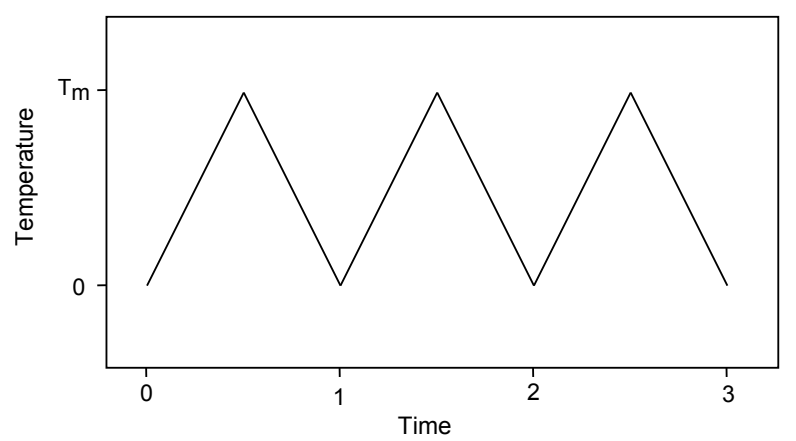

Figure 9: Evolution of temperature load with time for the bar. 


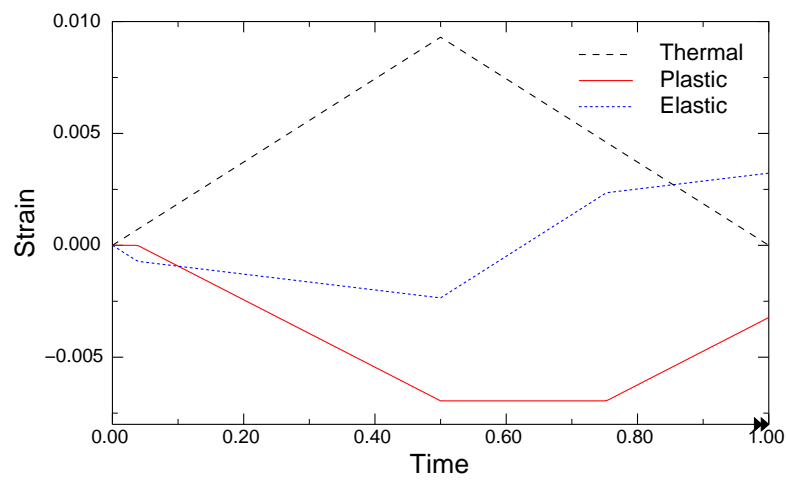

Figure 10: Evolution of different strain measures with time while using the temperature independent linear isotropic hardening model.

strain, the plastic strain stays constant. With the further decrease in the thermal strain, the material reaches its elastic limit and subsequently starts yielding in tension. This yielding in tension causes a reduction in the magnitude of plastic strain. At the end of the first load cycle as the thermal strain vanishes, residual elastic and plastic strains develop in the material. This causes residual stresses in the material even when the external source of excitation has been removed. Figure 11a shows the evolution of longitudinal stress versus the longitudinal plastic strain for the three cycles of temperature load. Residual tensile stress can be seen at the end of loading cycles.

The computed stresses and strains strongly depend on the material model used. In figures 11 and 12, the stress-strain graphs for the bar are shown with temperature independent and temperature dependent material models, receptively. By comparing the results it can be seen that relatively, the stresses are lower for the temperature dependent models, as compared to the temperature independent material models. This reduction in the stresses is attributed to the reduction in hardening of the material at high temperatures. Furthermore with the temperature dependent material models, the graphs show higher plastic strain which is attributed to larger thermal strain due to higher thermal expansion coefficient at higher temperatures.

The stress analysis results show that during a load cycle, with increasing temperatures, high compressive stress is generated, but when the material cools down and thermal strain vanishes, the compressive stresses transform to tensile stresses. This can be observed for all material models, but the magnitude of the residual stress is relatively lower with the kinematic hardening as compared to the isotropic hardening models. Furthermore, in the case of the kinematic hardening model, it can be seen that after the first cycle the stress-strain behavior becomes approximately stable. 


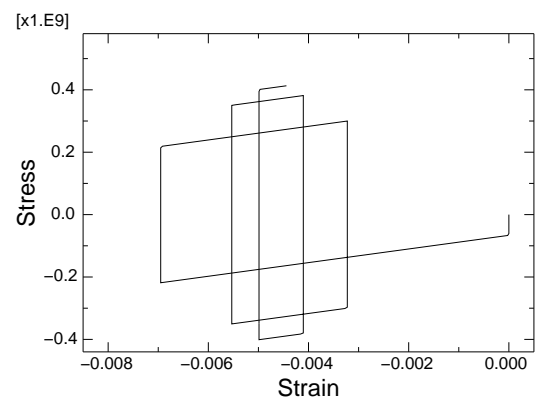

(a) linear isotropic hardening

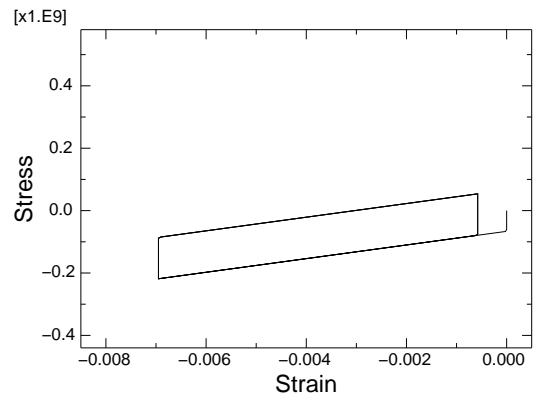

(b) linear kinematic hardening

Figure 11: Evolution of the longitudinal stress versus the longitudinal plastic strain for the bar, with temperature independent material models, subjected to the cyclic temperature variations.

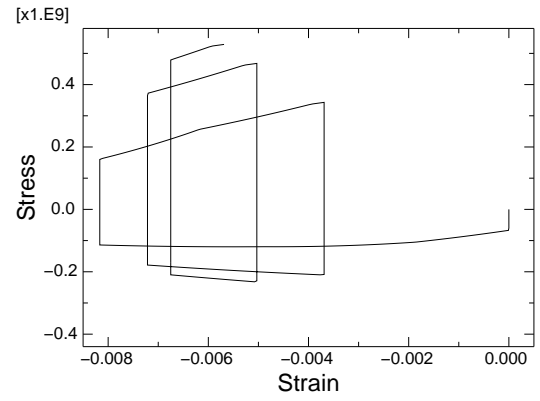

(a) linear isotropic hardening

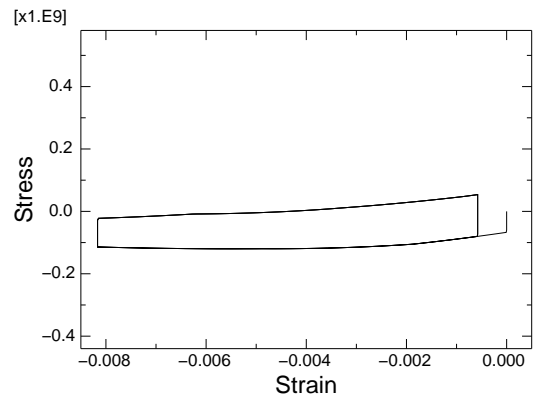

(b) linear kinematic hardening

Figure 12: Evolution of the longitudinal stress versus the longitudinal plastic strain for the bar, with temperature dependent material models, subjected to the cyclic temperature variations. 


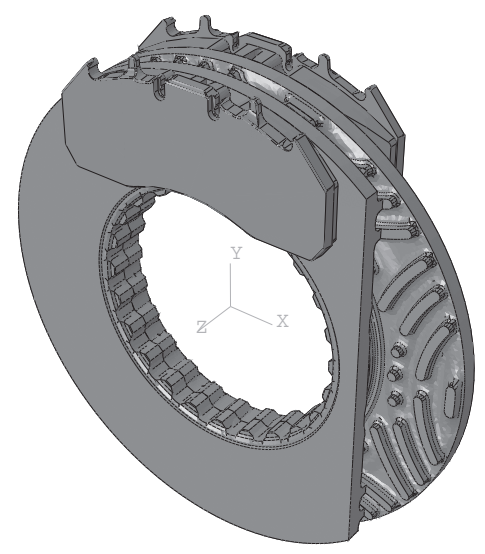

Figure 13: The assembly of the disc-pad system with a disc shown sectioned.

\subsection{Results for the disc-pad system}

The assembly of the disc-pad system considered in this work is shown in figure 13 with one disc sectioned to reveal the ventilation vanes (patented [30]). This is the assembly of a disc brake system of a heavy Volvo truck. In this hybrid or composite design, the mounting bell is not a part of the brake disc. The disc is geometrically symmetric about a plane normal to the z-axis. It is assumed that all thermomechanical loads applied to the disc are symmetric. Due to these reasons it could be assumed that coning or buckling does not take place. Therefore only a half of this assembly seems sufficient to be considered for the simulation.

The splines at the inner periphery of the disc are used to mount the disc to the wheel hub by engaging corresponding splines. For the simulation of thermal stresses these splines are not considered important so they have been removed to simplify the model. For the same reason, some geometry of the back plate has been removed. The assembly with simplified geometries of the disc and the back plate is shown in figure 14 .

Simulation of thermal stresses has been performed with the sequential approach. The results show that during hard braking, high compressive stresses are generated on the disc surface in circumferential direction which cause plastic yielding. But when the disc cools down, the compressive stresses transform to tensile stresses. Such results for a single braking operation have been presented in Paper II where the plasticity model is taken to be the von Mises yield criterion with nonlinear isotropic hardening, and both the hardening and the yield limit are temperature dependent.

For repeated braking it is important to use the kinematic hardening model as the isotropic hardening model cannot represent the Bauschinger effect. It has 


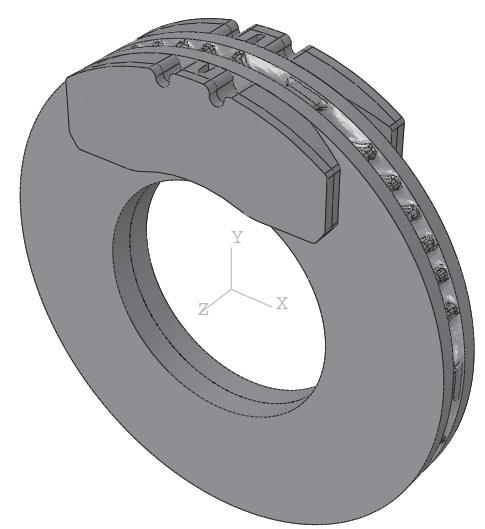

Figure 14: The assembly of the disc-pad system after removing the geometry not considered important for the simulation.

been shown in [31] that in grey cast iron, for a cyclic loading resulting in plastic deformation in both tension and compression, the kinematic hardening model gives a somewhat better agreement with experimental data than isotropic hardening.

In Paper III, results of an analysis for repeated braking are presented. Here the plasticity model is taken to be the von Mises yield criterion with linear kinematic hardening and both the hardening and the yield limit are temperature dependent. Figure 15 shows the temperature distribution on the disc surface after a brake application during this analysis. A ring of high temperatures, called hot band, can be distinguished in the middle of the disc surface. Figure 16 shows a ring in the middle of disc surface, at the end of brake application, with relatively higher compressive circumferential stresses which roughly corresponds to the ring of high temperatures. The disc is cooled after this braking operation, completing one brake cycle. It is assumed that braking conditions are same for all the brake cycles so that they generate similar temperature histories. Hence the temperature history generated during one brake cycle is merged three times in a sequence. In figure 17, graphs of circumferential stresses plotted against different measures of strain in circumferential direction, for three brake cycles, are given. The node chosen for these plots is located on the disc surface at $180^{\circ}$ from the middle of the pad and at a radius of 163.9 [mm]. It can be seen that residual tensile stresses in circumferential direction are predicted with both hardening models but with the kinematic hardening model these stresses are lower in magnitude as compared to the isotropic hardening model. After the first cycle an approximately stable stress-strain loop is obtained for the linear kinematic hardening model. So if the fatigue life data for the disc material is known, its fatigue life can be assessed. Furthermore, the results also show the appearance of tensile stresses in the radial direction during braking and cooling of the disc. But the residual radial stresses are compressive as compared to the residual circumferential stresses which are tensile. 


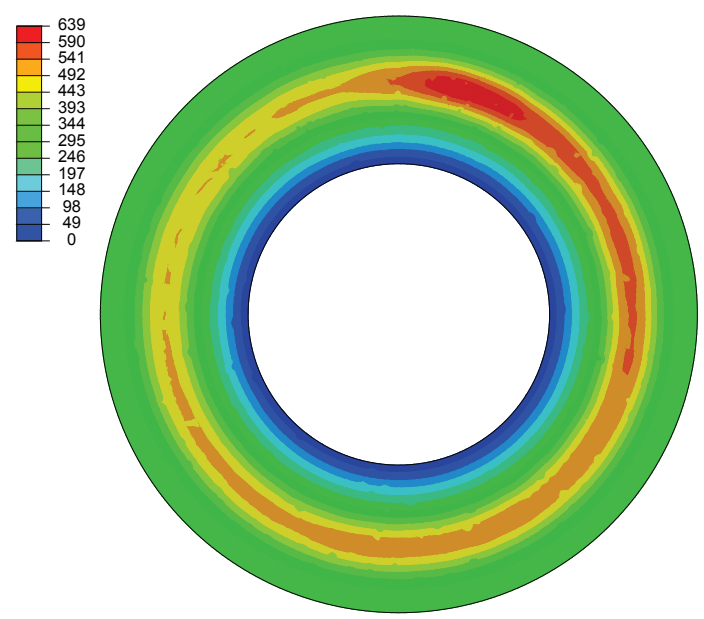

Figure 15: After the brake application, a ring of high temperatures develops on the disc surface.

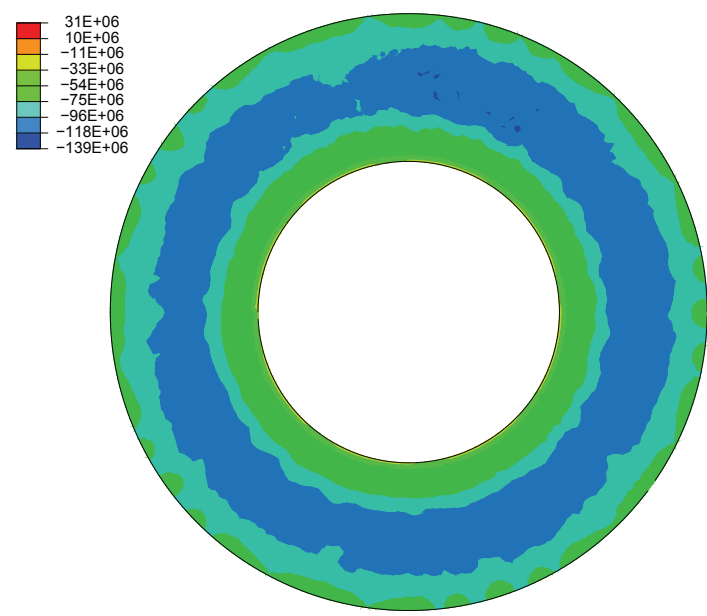

Figure 16: Circumferential stresses at the end of brake application during first cycle with the linear kinematic hardening model. 
This is indeed in agreement with the observation that radial microcracks on disc surfaces are more marked than circumferential ones, even when macroscopic cracks do not appear [10]. Figure 18 shows a ring in the middle of the disc surface, at the end of three brake cycles, where effective plastic strain is relatively higher. So the material in this area is most susceptible to fatigue cracks.

The simulation results presented in the first two papers predict one hot band in the middle of the disc. It has been explained by showing the contact pressure plots at different time steps. It is also shown (in Paper IV) that convex bending of the pad due to thermal deformations is the major cause of contact pressure concentration and hence appearance of hot bands. In the first two papers wear of the pad is not considered as it does not show much influence on the temperature distribution during a single braking operation for a pad without wear history and hence on the stresses.

The results show that when wear is considered, different distributions of temperature on the disc surface are obtained for each new brake cycle. After a few braking cycles two hot bands appear on the disc surface instead of only one, which is in agreement with experimental observation. The influence of wear on temperature distribution is discussed in Paper IV.

This sequential approach has proved very cheap in terms of computational time when compared to a fully coupled Lagrangian approach. Significantly lower computational resources required to simulate a disc brake by using the sequential approach gives the freedom to perform multi-objective optimization studies. Such a study is performed in Paper $\mathrm{V}$ where the mass of the back plate, the brake energy and the maximum temperature generated on the disc surface during hard braking are optimized. The design variables are the applied load of braking, Young's modulus of friction material and the thickness of back plate. The results indicate that a brake pad with lowest possible stiffness will result in an optimized solution with regards to all three objectives. The results also reveal a linear relation of applied braking load and brake energy. Another interesting result is the trend of a decrease in maximum temperature with an increase in back plate thickness. 


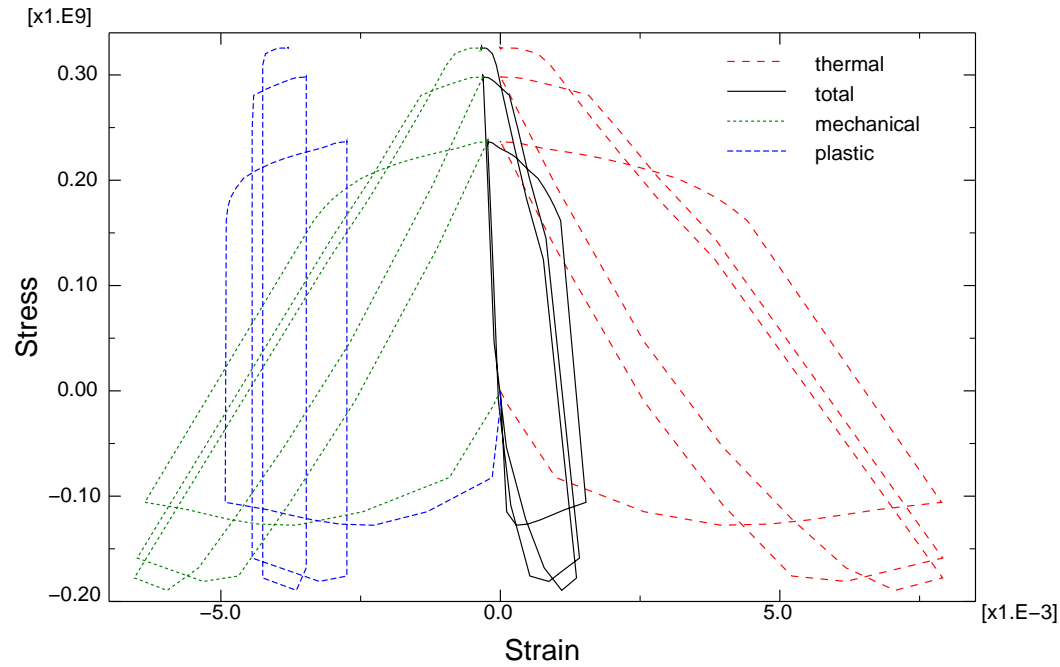

(a) linear isotropic hardening

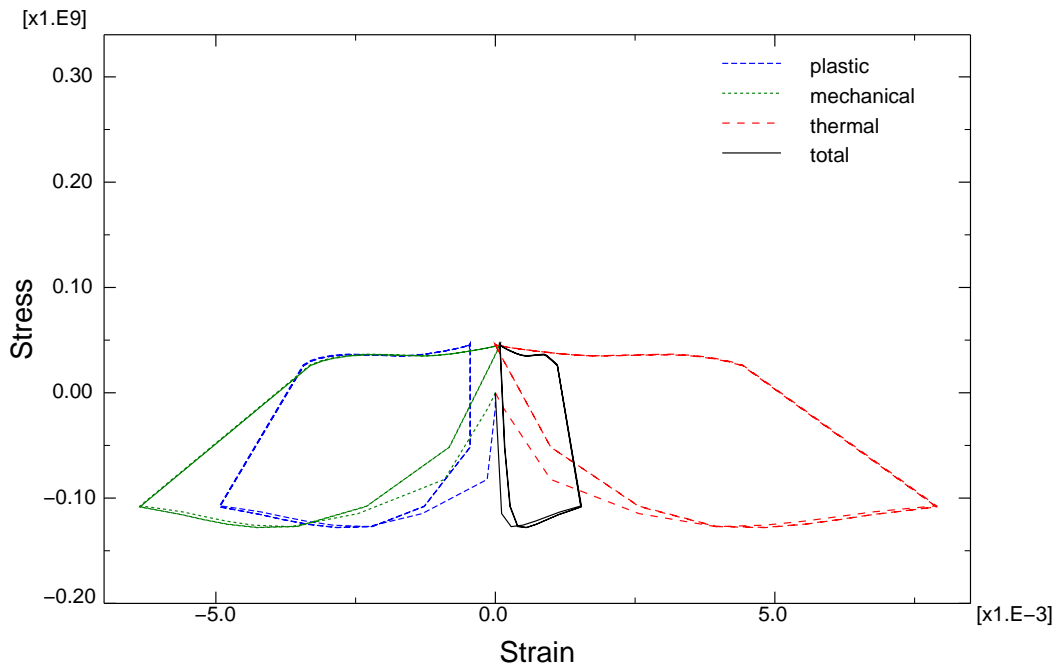

(b) linear kinematic hardening

Figure 17: Evolution of the circumferential stress versus the circumferential strain for the repeated braking. 


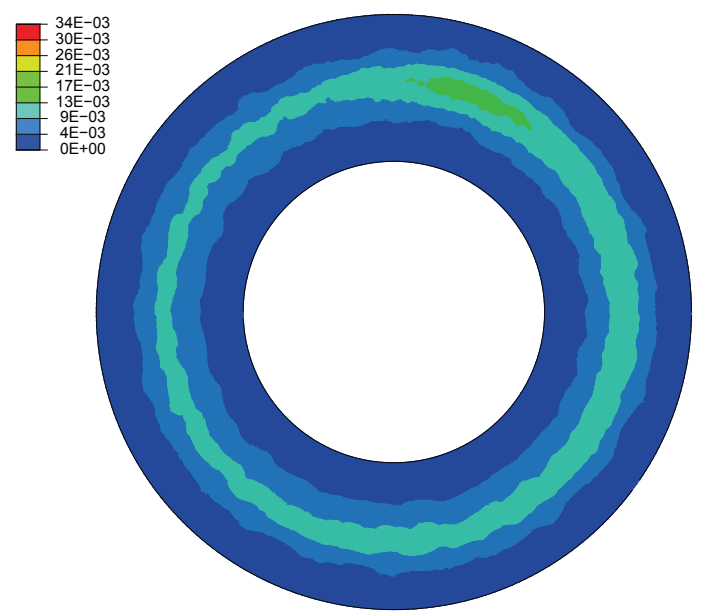

Figure 18: Effective plastic strain at the end of third brake cycle with the linear kinematic hardening model. 


\section{Prosthetic sockets}

\subsection{Background}

A prosthetic socket acts as an interface between a subject's amputated limb and prosthesis. Its main purpose is to provide a comfortable interface by distributing of load over the residual limb. Biomechanical understanding of the interaction between the soft tissues and the prosthetic socket is very important in order to design a comfortable and functional socket. The contact pressure distribution at the socket-limb interface has been a critical consideration for the practice of socket design [32].

Three different concepts of socket designs [33] are commonly used in lower limb amputees: total contact (TC), total surface-bearing (TSB) and hydrostatic (HS) socket. The geometries of the TC, TSB and HS sockets considered in this work are shown in figure 19. In a TC socket, the basic idea is to distribute the load over load tolerant areas while relieving the sensitive areas of the residual limb. At the same time a total contact at the distal end of the limb is aimed. In a TSB socket, there are no relieving areas but higher load is targeted at load tolerant areas. The aim in this socket design is that weight is carried by the entire surface of the residual limb. The HS socket concept aims to apply pressure equally over the limb and assumes that the tissues behave as a fluid at rest, so that any additional pressure will be transmitted equally to every point in the fluid. Liners or socks may be used in between the skin and the socket but are not considered in this work.

Despite the improved designs of prosthetic sockets, skin problems are reported by a considerable number of lower limb amputees [34]. Pressure ulcer is considered the most common skin problem. In addition to the superficial skin problems, a subject using a prosthetic socket can also suffer from deep tissue injury (DTI) [35]. Thus, it is also important to investigate the internal mechanical conditions in the soft tissues while considering a socket design.

FE models have been used to investigate the contact pressure at the skin-socket interface in an effort to optimize the design of a prosthetic socket while ignoring the internal mechanical conditions in the limb, see e.g. [36, 32]. Recently, see e.g. [37], [38], [39] and [40], internal mechanical conditions in the lower limb under external compression have been investigated. One concern about the previous studies 


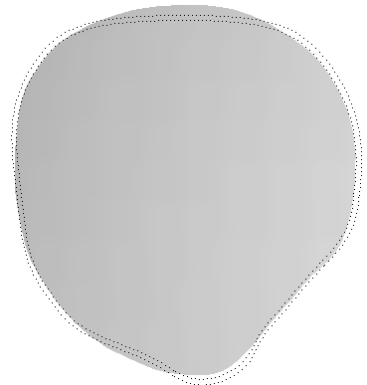

(a) TC

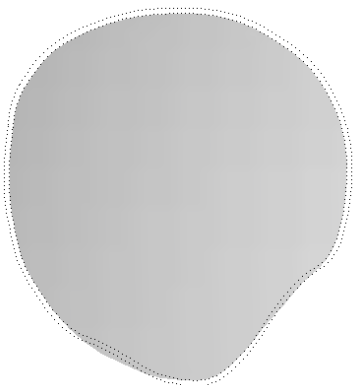

(b) TSB

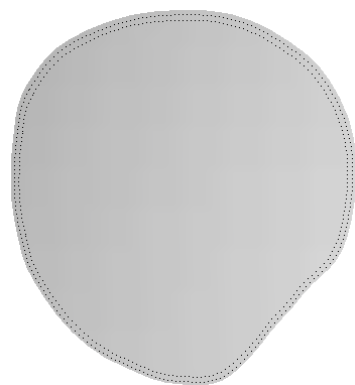

(c) HS

Figure 19: Cross-section of the total contact (TC), total surface-bearing (TSB) and hydrostatic (HS) sockets shown in relation to limb cross-section. Dotted lines represent the socket while the limb cross-section is shown in grey color bounded by solid lines.

is that soft tissues have been lumped together to simplify the model. Another concern is that in these works, displacements or contact pressure have been determined by physical experiments and used as a boundary condition in numerical computations. Although such studies give valuable insight, for the design practice of prosthetic sockets, contact analysis should be performed to determine the boundary conditions.

In this work, the interface stresses and internal mechanical conditions in a lower limb for three different socket designs are compared. A detailed FE model of a transtibial cross-section is developed. Skin, fat, fascia, muscles, large blood vessels and bones are represented separately, which is novel in this research. The large blood vessels are represented as a linear elastic material. Material properties for the soft tissues are obtained from literature. The contact problem is solved between socket and limb while taking the friction into consideration to determine the contact pressure and resultant internal stress-strain in the soft tissue. As can be seen in figure 19, initially, the socket and the limb cross-section overlap which require the contact algorithm to resolve the overclosure gradually over multiple increments. Influence of blood pressure is also explored by using different moduli of elasticity for the blood vessels.

The results show that the internal mechanical conditions are significantly different when separate material models are used for the soft tissues as compared to when the tissues are lumped. The results also highlight that blood pressure could have a significant influence on the internal mechanical conditions. Detailed results for this study are presented in Paper VII. 


\section{Review of included papers}

\section{Paper I}

In this paper a new stabilized augmented Lagrangian multiplier method is implemented for the finite element solution of contact problems. The method is implemented for a unilateral contact problem. Small deformations and frictionless contact is assumed.

\section{Paper II}

In this paper results of a simulation of stresses in a brake disc for a single braking operation are presented. The plasticity model is taken to be the von Mises yield criterion with nonlinear isotropic hardening, where both the hardening and the yield limit are temperature dependent.

\section{Paper III}

In this paper results of a simulation of thermal stresses in a brake disc for repeated braking are presented. The plasticity model is taken to be the von Mises yield criterion with linear kinematic hardening, where both the hardening and the yield limit are temperature dependent.

\section{Paper IV}

In this paper the influence of the wear history of a pad on the temperature distribution on a disc surface is presented. It is also shown that convex bending of a pad assembly as a result of thermal deformations is a significant factor towards the concentration of contact pressure in the middle of a pad. 


\section{Paper V}

In this paper results for a multi-objective optimization of a disc brake system are presented. The mass of the back plate of the brake pad, the brake energy and the maximum temperature generated in the disc during hard braking are optimized. The design variables are the applied load of braking, Young's modulus of friction material and the thickness of the back plate.

\section{Paper VI}

In this paper a literature review of disc brakes and related phenomena is presented. A detailed description of different geometries and materials for the components of a brake assembly is given. The evolution of tribological interface of disc-pad system is also covered in detail here. Different operational problems such as fade, geometrical deviations and noise are also discussed.

\section{Paper VII}

In this paper, interface pressure and internal mechanical conditions in a lower limb donned in a prosthetic socket are investigated. Skin, fat, fascia, muscles, large blood vessels and bones are represented separately and material properties for them are obtained from literature. The contact interaction between the skin and the socket also includes friction. Comparison of the internal mechanical conditions and interface stresses for three different socket designs is also presented. 


\section{Bibliography}

[1] A. Landenberger and A. El-Zafrany. Boundary element analysis of elastic contact problems using gap finite elements. Computers and Structures, 71(6):651$661,1999$.

[2] F. Chouly and P. Hild. A nitsche-based method for unilateral contact problems: Numerical analysis. SIAM Journal on Numerical Analysis, 51(2):12951307, 2013.

[3] T.A. Laursen. Computational Contact and Impact Mechanics: Fundamentals of Modeling Interfacial Phenomena in Nonlinear Finite Element Analysis. Springer Berlin Heidelberg, 2003.

[4] G. Zavarise and P. Wriggers, editors. Trends in Computational Contact Mechanics. Springer Berlin Heidelberg, 2011.

[5] P. Wriggers. Computational Contact Mechanics. Springer Berlin Heidelberg, 2006.

[6] P. Heintz and P. Hansbo. Stabilized lagrange multiplier methods for bilateral elastic contact with friction. Computer Methods in Applied Mechanics and Engineering, 195(33-36):4323-4333, 2006.

[7] T.K. Kao, J.W. Richmond, and A. Douarre. Brake disc hot spotting and thermal judder: an experimental and finite element study. International Journal of Vehicle Design, 23(3):276-296, 2000.

[8] D. Eggleston. An investigation into frictional surface interactions and their effect on brake judder. PhD thesis, Sheffield Hallam University, 2000.

[9] S. Koetniyom, P.C. Brooks, and D.C. Barton. The development of a material model for cast iron that can be used for brake system analysis. Proceedings of the Institution of Mechanical Engineers, Part D: Journal of Automobile Engineering, 216(5):349-362, 2002.

[10] P. Dufrénoy and D. Weichert. A thermomechanical model for the analysis of disc brake fracture mechanisms. Journal of Thermal Stresses, 26(8):815-828, 2003.

[11] S. Panier, P. Dufrénoy, and D. Weichert. An experimental investigation of hot spots in railway disc brakes. Wear, 256(7-8):764-773, 2004. 
[12] S. Panier, P. Dufrénoy, J.F. Brunel, and D. Weichert. Progressive waviness distortion: A new approach of hot spotting in disc brakes. Journal of Thermal Stresses, 28(1):47-62, 2005.

[13] S. Koetniyom. Thermal Stress Analysis of Automotive Disc Brakes. PhD thesis, University of Leeds, 2000.

[14] T. Deichmann and H. Lathwesen. Sheet cast disc-brake disc innovation through functional integration. Proceedings of the Eurobrake, 16-18 April, Dresden, Germany, 2012.

[15] A. Giorgetti. Disc for a disc brake for vehicles in general and for highperformance cars in particular, November 28 2000. US Patent 6,152,270.

[16] D.J. Kim, Y.M. Lee, J.S. Park, and C.S. Seok. Thermal stress analysis for a disk brake of railway vehicles with consideration of the pressure distribution on a frictional surface. Materials Science and Engineering: A, 483-484(1-2 C):456-459, 2008.

[17] F. Bagnoli, F. Dolce, and M. Bernabei. Thermal fatigue cracks of fire fighting vehicles gray iron brake discs. Engineering Failure Analysis, 16(1):152-163, 2009 .

[18] C. Gao, J.M. Huang, X.Z. Lin, and X.S.C. Tang. Stress analysis of thermal fatigue fracture of brake disks based on thermomechanical coupling. Journal of Tribology, 129(3):536-543, 2007.

[19] T.J. Mackin, S.C. Noe, K.J. Ball, B.C. Bedell, et al. Thermal cracking in disc brakes. Engineering Failure Analysis, 9(1):63-76, 2002.

[20] P. Dufrénoy and D. Weichert. Prediction of railway disc brake temperatures taking the bearing surface variations into account. Proceedings of the Institution of Mechanical Engineers, Part F: Journal of Rail and Rapid Transit, 209(2):67-76, 1995.

[21] J. Choi and I. Lee. Finite element analysis of transient thermoelastic behaviors in disk brakes. Wear, 257(1-2):47-58, 2004.

[22] K. Lee and J.R. Barber. An experimental investigation of frictionally-excited thermoelastic instability in automotive disk brakes under a drag brake application. Journal of Tribology, 116(3):409-414, 1994.

[23] M. Eriksson, F. Bergman, and S. Jacobson. On the nature of tribological contact in automotive brakes. Wear, 252(1-2):26-36, 2002.

[24] P. Dufrénoy. Two-/three-dimensional hybrid model of the thermomechanical behaviour of disc brakes. Proceedings of the Institution of Mechanical Engineers, Part F: Journal of Rail and Rapid Transit, 218(1):17-30, 2004.

[25] N. Strömberg. Development and implementation of an Eulerian aproach for efficient simulation of frictional heating in sliding contacts. In IV International 
Conference on Computational methods for Coupled Problems in Science and Engineering (ECCOMAS), Kos, Greece, 20-22 June 2011.

[26] N. Strömberg. An Eulerian approach for simulating frictional heating in discpad systems. European Journal of Mechanics - A/Solids, 30(5):673-683, 2011.

[27] S. Medonos. Study of structural behaviour of ventilated brake disc. SAE Technical Paper 831316, 1983.

[28] A.S. Khan and S. Huang. Continuum Theory of Plasticity. Wiley-Interscience, 1995.

[29] E. Gustafsson, M. Hofwing, and N. Strömberg. Residual stresses in a stress lattice-experiments and finite element simulations. Journal of Materials Processing Technology, 209(9):4320-4328, 2009.

[30] J. Hulten and I. Dagh. Brake disc for a vehicle disc brake, August 292006. US Patent 7,097,010.

[31] B.L. Josefson, U. Stigh, and H.E. Hjelm. Nonlinear kinematic hardening model for elastoplastic deformations in grey cast iron. Journal of Engineering Materials and Technology, Transactions of the ASME, 117(2):145-150, 1995.

[32] A.F.T. Mak, M. Zhang, and D.A. Boone. State-of-the-art research in lowerlimb prosthetic biomechanics-socket interface: A review. Journal of Rehabilitation Research and Development, 38(2):161-173, 2001.

[33] J. Fergason and D.G. Smith. Socket considerations for the patient with a transtibial amputation. Clinical Orthopaedics and Related Research, (361):7684, 1999 .

[34] K.M. Bui, G.J. Raugi, V.Q. Nguyen, and G.E. Reiber. Skin problems in individuals with lower-limb loss: Literature review and proposed classification system. Journal of Rehabilitation Research and Development, 46(9):10851090, 2009.

[35] A. Gefen. Deep tissue injury from a bioengineering point of view. Ostomy Wound Management, 55(4):26-36, 2009.

[36] M. Zhang, A.F.T. Mak, and V.C. Roberts. Finite element modelling of a residual lower-limb in a prosthetic socket: A survey of the development in the first decade. Medical Engineering and Physics, 20(5):360-373, 1998.

[37] Yarnitzky G. Yizhar Z. Kristal A. Oppenheim U. Siev-Ner I. Gefen A. Portnoy, S. Real-time patient-specific finite element analysis of internal stresses in the soft tissues of a residual limb: A new tool for prosthetic fitting. Annals of Biomedical Engineering, 35(1):120-135, 2007.

[38] Yizhar Z. Shabshin N. Itzchak Y. Kristal A. Dotan-Marom Y. Siev-Ner I. Gefen A. Portnoy, S. Internal mechanical conditions in the soft tissues of a 
residual limb of a trans-tibial amputee. Journal of Biomechanics, 41(9):18971909, 2008.

[39] Siev-Ner I. Shabshin N. Kristal A. Yizhar Z.-Gefen A. Portnoy, S. Patientspecific analyses of deep tissue loads post transtibial amputation in residual limbs of multiple prosthetic users. Journal of Biomechanics, 42(16):2686-2693, 2009 .

[40] Y. Wang, S. Downie, N. Wood, D. Firmin, and X.Y. Xu. Finite element analysis of the deformation of deep veins in the lower limb under external compression. Medical Engineering and Physics, 35(4):515-523, 2013. 


\section{Papers}

The articles associated with this thesis have been removed for copyright reasons. For more details about these see:

http://urn.kb.se/resolve?urn=urn:nbn:se:liu:diva-124572 